\title{
Erratum: Mechanical properties of graphene and boronitrene [Phys. Rev. B 85, 125428 (2012)]
}

\author{
R. C. Andrew, R. E. Mapasha, A. M. Ukpong, and N. Chetty \\ Q (Received 19 October 2019; published 5 November 2019)
}

DOI: 10.1103/PhysRevB.100.209901

Equation (10) for the two-dimensional bulk modulus $\gamma$ of a general anisotropic medium should be replaced by [1]

$$
\gamma=\left(c_{11} c_{22}-c_{12}^{2}\right) /\left(c_{11}+c_{22}-2 c_{12}\right) \text {. }
$$

Equation (10) was not used in our paper since all materials described were isotropic and since this expression is correct in the special case of isotropy. Thus, no results have been affected by this finding.

We acknowledge Prof. D. Tomanek and Prof. A. Every for pointing out the error.

[1] D. Liu, C. Daniels, V. Meunier, A. G. Every, and D. Tománek, In-plane breathing and shear modes in low-dimensional nanostructures, Carbon 157, 364 (2020). 